\title{
MEDIENWIRKSAME NEOLOGISMEN IN DER DEUTSCHEN GEGENWARTSSPRACHE ${ }^{1}$
}

\section{EINLEITUNG}

Neue oder veränderte Gegebenheiten in der außersprachlichen Realität, neue Sachverhalte, Arbeitsbereiche, Wissensgebiete und Konzepte verlangen entsprechende Veränderungen in der Sprache, welche die weltliche Realität sowie die geistige Welt der Sprachbenutzer widerspiegelt. Es ist eine Tatsache, daß gerade der Wortschatz diejenige Komponente der Sprache ist, die den ständigen Veränderungen und Wandlungsprozessen am meisten unterliegt und daß diese in immer wieder neuen kognitiven und kommunikativen Bedürfnissen einer Sprachgemeinschaft begründet sind. Es entstehen zahlreiche neue Wörter - Neologismen, die als lexikalische Entsprechungen veränderter außersprachlicher Bedingungen der Kommunikation zu interpretieren sind.

Die untersuchten Neologismen entstammen der deutschen überregionalen Tagesund Wochenpresse. Für eine solche Auswahl spricht ein einleuchtender Grund: Die Presse wird nämlich als ein Bereich des öffentlichen Sprachgebrauchs mit zahlreichem Publikum verstanden. Sie dokumentiert den jeweiligen Sprachzustand und spielt bei der Ausprägung bzw. Veränderung sprachlicher Normen eine wichtige Rolle. Ihr massenmedialer Charakter gibt ihr die Möglichkeit, die Sprachentwicklung zu beeinflussen und sogar zu steuern; ihre allgemeine Zugänglichkeit, ihre hohen Auflagen und periodischen Erscheinungsweisen begünstigen die potentiellen Auswirkungen des Sprachgebrauchs in der Presse auf die sprachliche Norm. Die Relevanz der Presse im Prozeß der Sprachentwicklung betont MOSER $(1959,446)$ wie folgt:

"Die Zeitung übernimmt weithin die frühere Funktion der Dichtung. Sie vermittelt zusammen mit dem populärwissenschaftlichen Buch der allgemeinen Hochsprache die Neuerungen des Wortschatzes, auch solche fachsprachlicher Art; bis zu einem gewissen Grad ist die Zeitungssprache auch selbst sprachschöpferisch."

1 Der vorliegende Beitrag ist im wesentlichen eine Zusammenfassung der gleichnamigen Magisterarbeit, die am 15.7.1994 an der Philosophischen Fakultät Ljubljana vor der Kommission Prof. Dr. Siegfried Heusinger (Mentor), Prof. Dr. Mirko Križman und Prof. Dr. Stojan Bračič verteidigt wurde. Die theoretische Diskussion über den Neologismus-Begriff sowie das Kapitel über die Wortbildungsstruktur der untersuchten Neologismen werden hier jedoch nicht berücksichtigt. 
Die Pressesprache scheint dementsprechend für lexikalische Untersuchungen besonders angebracht $z u$ sein. LÜGER $(1983,30)$ stellt fest, daß sie nicht etwa nur bis zu einem gewissen Grad sprachschöpferisch ist, sondern daß die Verwendung neuer Lexik in der Pressesprache besonders umfangreich und auffallend ist. Die Ursachen dafür liegen in ständigen Veränderungen sozialer, technischer und wissenschaftlicher Verhältisse, welche in der veränderten bzw. neuen Lexik ihre Widerspiegelungen finden. Das zeigt sich vor allem in den Massenmedien, deren Hauptfunktion ja darin besteht, das Neue, noch nicht Bekannte zu übermitteln. Es wird daher angenommen, $\mathrm{da} B$ der Sprachgebrauch in der Presse als ein Indiz für lexikalische Entwicklungstendenzen in der Gegenwartssprache (und darunter besonders für die Entstehung und Verwendung neuer Lexik) gelten kann.

Es wurden zur Analyse drei deutsche überregionale Zeitungen herangezogen, und zwar: die Frankfurter Allgemeine Zeitung (FAZ), die Süddeutsche Zeitung (SüdZ) und Die Zeit $(\mathbf{Z})$. Von allen drei Zeitungstiteln wurde jeweils eine Samstagsausgabe ausgewertet; insgesamt wurden 163 Zeitungsseiten untersucht. Textsorten wie Anzeige, Stellenangebot, Börsenspiegel, Wetterbericht, Zeitungsroman, Fernseh- und Bühnenprogramm wurden nicht analysiert Auf diese Weise entstand eine Neologismensammlung von 456 jeweils zusammen mit sprachlichem Kontext exzerpierten Wortbeispielen. Die meisten fehlen in den gängigen Wörterbüchern, alle entsprechen aber dem Begriff Neologismus, so wie er in der vorliegenden Untersuchung verstanden und definiert wird.

Die Wahl dreier überregional verbreiteter Zeitungen möge eine gewisse Homogenität des untersuchten Materials gewährleisten, denn der Sprachgebrauch in verschiedenen Zeitungstypen (z.B. in der Boulevardpresse oder in der lokalen/regionalen Presse) unterscheidet sich wesentlich voneinander. Die ausgewählten Zeitungen gehören zum Typ der Abonnementzeitung an und richten sich an ein relativ homogenes Publikum, an eine "gebildete Leserschaft" (MEYER 1974, 99). Sie sind weit verbreitet und nicht lokal bzw. regional begrenzt; lokale bzw. regionale sprachliche Besonderheiten werden damit einigermaßen vermieden. Es muß aber betont werden, daß auch im Rahmen einer einzigen Zeitungsart eine gewisse sprachliche Heterogenität vorliegt. Unterschiedliche Rubriken, in denen viele Lebensbereiche sprachlich realisiert werden, beeinflussen die Auswahl unterschiedlicher Darstellungsformen (Textsorten), welche einen charakteristischen Sprachgebrauch aufweisen. Die jeweilige Textsortenauswahl hängt wiederum von den unterschiedlichen Erscheinungsweisen der Presse an. Bei der Betrachtung der Pressesprache ist demnach die Berücksichtigung der Produktions- und Rezeptionsbedingungen von Texten von großer Bedeutung, da sprachliche Äußerungen in der Regel Teile bestimmter kommunikativen Prozesse sind und da sie im Rahmen konkreter sozialen Situationen realisiert werden (vgl. LÜGER 1983, 46). Die Lexik ist demgemäß nicht als eine isolierte sprachliche Erscheinung zu beobachten, sondern immer im Zusammenhang mit zahlreichen Faktoren, die die jeweilige Wortauswahl im Prozeß der Textgestaltung wesentlich mitbestimmen. Die 
Wörter werden den Kommunikationsbedingungen und -absichten entsprechend gebraucht (in unserem Zusammenhang auch neu gebildet). Neben den grammatischen und semantischen Eigenschaften sollen demnach auch pragmatische Eigenschaften eines neuen Wortes berücksichtigt werden. Es ist wichtig, in welchen Situationen, unter welchen Bedingungen, gegenüber welchen Kommunikationspartnern kann/darf ein Wort gebraucht werden, welche Intentionen werden damit realisiert und welche Wirkungen erzielt.

Die Wirkungen der Massenkommunikation werden als "Veränderungen im Verhalten und Erleben, die durch die Zuwendung zu Massenmedien und durch das Aufnehmen von Aussagen der Massenkommunikation 'bewirkt' werden" verstanden (MALETZKE 1976, 181). Sie sind Ergebnisse komplexer Wechselbeziehungen zwischen den zahlreichen an der Massenkommunikation beteiligten Faktoren. ${ }^{2}$

So werden auch die Neologismen in der vorliegenden Untersuchung als medienwirksam verstanden. Massenmedial verwendet bewirken sie wegen ihrer Neuheit Reflexion beim Leser. Man denkt über den bekannten Wortschatz gewöhnlich nicht nach; die Überlegung beginnt erst dann, wenn man einem bisher noch unbekannten bzw. noch nicht geläufigen Wort oder einer neuen Wortbedeutung begegnet (vgl. MÜLLER 1976, 867). Es wird außerdem angenommen, daß der Gebrauch eines Wortes in unerwarteten, ungewöhnlichen Umgebungen bzw. in "neuen" Kommunikationsbereichen beim Rezipienten ebenso neu bzw. auffällig wirken kann. Die Wirkung der in der Presse verwendeten Neologismen zeigt sich aber nicht nur in ihrer Neuheit und daher ihrer Auffälligkeit; sie zeigt sich auch darin, daß sie Einstellungen und Verhaltensweisen beim Leser mitbestimmen kann (vgl. SCHMITZ 1987, 821).

Der Neologismengebrauch in der Presse soll demgemäß unter unterschiedlichen, eng miteinander verbundenen Aspekten untersucht werden. Die Struktur, Funktion und Wirkung von Neologismen sollen zu gleichwertigen Kategorien der lexikalischen Untersuchungen im Bereich der lexikalischen Innovation werden.

\section{DER BEGRIFF DES NEOLOGISMUS}

Der Terminus Neologismus stammt aus dem Griechischen: neos bedeutet neu und logos ist Wort. Er ist ein neues Wort, eine lexikalische Neuerung, eine lexikalische In-

2 G. MALETZKE (1976, 181ff.) berichtet ausführlich über die an der Massenkommunikation beteiligten Faktoren, die die Wirkungsprozesse mitbestimmen. Im Rahmen eines Wirkungsmodells der Massenkommunikation stellt er folgende Phasen des Wirkungsprozesses fest: das Angebot von Medienaussagen, die subjektive und objektive Situation des Rezipienten, der dem Angebot gegenübersteht, die personalen, objektbezogenen und habituellen Prädispositionen, die der Rezipient in die Situation mitbringt und die psychischen Prozesse, welche sich beim Rezipienten in der Begegnung mit den Aussagen abspielen und welche schließlich zu den eigentlichen Wirkungen führen. 
novation in der Sprache, die zur bestimmten Zeit bei der Sprachgemeinschaft als neu und daher nicht allgemein bekannt empfunden wird.

Der Bedarf an neuer Lexik kann in formaler Hinsicht unterschiedlich befriedigt werden. Die wichtigsten Prozesse, die der Entstehung neuer Lexik zugrunde liegen, sind Wortschöpfung, Wortbildung, Entlehnung und Bedeutungsveränderung. Für die vorliegende Untersuchung scheinen nur die letzten drei relevant zu sein, während die Wortschöpfung als eine "erstmalige Zuordnung eines Lautkomplexes zu einer Bedeutung" (SCHIPPAN, 1992, 107) für uns ohne Belang ist. Heute wird der Wortschatz auf diese Weise relativ selten erweitert, und die Wortschöpfung ist etwa auf Onomatopoetica und Kindersprache begrenzt bzw. charakteristisch für die Bildung neuer Eigennamen in der Werbung.

Die bisherige theoretische Beschäftigung mit dem Phänomen Neologismus weist verschiedene Auffassungen zu dieser lexikalischen Kategorie nach. Die unterschiedlichen Wege, die zur Entstehung von Neologismen führen können, zeigen sich auch in zahlreicher und nicht selten uneinheitlich definierter Terminologie, die in bezug auf den Neologismus gebräuchlich ist: Neologismus, Neuwort, Neubildung, Neuprägung, Neuschöpfung, Neubedeutung, Neosemantismus, Neulexem, Neusemem, Neuformativ, Okkasionalismus. Unter Berücksichtigung der relevanten Literatur zum Thema gelten in der vorliegenden Untersuchung folgende Abgrenzungskriterien der Neologismen von den übrigen Wörtern:

- Neologismen sind diejenigen Wörter, die beim Sprachbenutzer als neu und daher meist als auffällig empfunden werden.

- Sie sind neue Wortbildungskonstruktionen, d.h. Neubildungen oder neue Bedeutungen eines vorhandenen Wortes, d.h. Neubedeutungen.

- Sie sind entweder einheimische oder entlehnte Wörter; bei den Wortbildungskonstruktionen können sie deren Kombinationen sein.

- Sie gehören unterschiedlichen Sprachschichten an; sie können ihre sprachliche Varietät und ihren Kommunikationsbereich wechseln.

- Ihre Entstehungszeit liegt in der jüngsten Vergangenheit, und die Mehrzahl der Sprachgemeinschaft betrachtet sie (noch) nicht als festen Bestandteil ihres Wortschatzes. Die Lexikalisierung hat (meist) noch nicht stattgefunden; sie sind in den Wörterbüchern meist (noch) nicht kodifiziert. Es folgt demnach, $\mathrm{daß}$ zu den Neologismen auch gelegentliche Bildungen (Okkasionalismen) gezählt werden. 


\section{NEOLOGISMEN HINSICHTLICH IHRER WORTARTENZUGEHÖRIGKEIT}

Es wurden drei Hauptwortarten des Deutschen beobachtet: Substantiv, Adjektiv und Verb. Diese machen auch den Hauptteil des Gesamtwortschatzes aus: Über die Hälfte des Wortbestandes im Deutschen sind Substantive; ein Fünftel davon sind Verben und die drittgrößte Wortart im Deutschen sind Adjektive. ${ }^{3}$

Es ist festzustellen, daß Substantive die meist vertretene Wortart in der untersuchten Neologismensammlung sind: 427 Substantive machen 93,6\% des Korpus aus. An der zweiten Stelle stehen Adjektive mit 21 Belegen (4,6\%) und Verben sind mit 8 Beispielen vertreten $(1,8 \%)$.

Der Anteil an Substantiven ist sogar höher als z.B. ihr Anteil an neuen Wörtern bei einer Neuauflage des Dudens. Ein Vergleich der 16. Auflage des Mannheimer Dudens mit der vorhergehenden ermittelte nämlich eine Zunahme von $7 \%$ neuer Wörter und $80,4 \%$ davon waren Substantive (SOMMERFELDT 1988, 115).

Wegen der absoluten Dominanz der Substantive konzentriere ich mich im folgenden nur auf diese Wortart und versuche festzustellen, wodurch diese Tatsache zu begründen ist.

Die statistisch bewiesene Häufung von Substantiven verifiziert das wesentliche und auffällige Merkmal der deutschen Gegenwartssprache, den Nominalstil. Es geht dabei um Überwiegen der Substantive und eventuell auch der Adjektive, wobei die Verben an ihrer semantischen Bedeutung verlieren können und nicht selten nur ihre syntaktische Rolle als Prädikat ausüben. Da die substantivischen Satzglieder weit ausgebaut und attribuiert werden können, nimmt die Anzahl der Substantive in Texten wesentlich zu.

Die hohe Frequenz von Substantiven ist aber auch rein syntaktisch bedingt. Generell gilt ja, daß jeder Satz mehrere Nomina enthalten kann, dagegen aber nur ein finites Verb als Prädikat. Daher genügen die rein quantitativen Angaben nicht, um den Nominalstil festzustellen oder sogar zu bewerten.

Bei der Betrachtung des exzerpierten Wortschatzes haben sich drei Hauptgruppen von Gründen und Ursachen für die Dominanz der Substantive herausgestellt. Erstens unterstützen sie das für die Presse charakteristische Streben nach konzentrierter Information. Die Ursachen dafür mögen demnach auch in den medienspezifischen Eigenschaften der Presse liegen. Darunter werden Eigenschaften der Zeitung als Me-

DEUTSCHE SPRACHE. Kleine Enzyklopädie. 1983, 154, 145, 159. Vgl. dazu auch DUDEN Grammatik der deutschen Gegenwartssprache. 1984, 91; H. BUBMANN: Lexikon der Sprachwissenschaft. 1990, 752; W. ORTMANN: Hochfrequente deutsche Wortformen, Bd. III. 1979. Ortmann gibt folgende Angaben über die Frequenz der Hauptwortarten im Deutschen an: Substantive 46,9\%, Adjektive 22,6\% und Verben $19,3 \%$ (XXXI). 
dium verstanden sowie auch Eigenschaften bestimmter Textsorten der Tages- bzw. Wochenpresse. Die Presse strebt danach - zumindest gilt das für kürzere und informationsbetonte Textsorten - möglichst viele Informationen auf engem Raum zusammenzufassen. Der begrenzte Raum, der zur Verfügung steht, zwingt zur Kürze. Es kann daher angenommen werden, daß Nominalisierungen in der Presse auch aus Gründen der Informationsverdichtung erfolgen: Sie sind sprachliche Folgen bestimmter medienspezifischen Eigenschaften. In bezug auf die Wortbildung zeigen sich lexikalische Verdichtungen von Informationen in der Pressesprache in einem überaus häufigen Vorkommen von Komposita (vgl. LÜGER 1983, 17). Die hohe Aktivität und Produktivität der Komposition im substantivischen Bereich des Deutschen werden auch bei FLEISCHER/BARZ $(1992,84)$ festgestellt. Die Möglichkeiten für die Bildung neuer Komposita scheinen nur wenig begrenzt zu sein. Auch die statistische Auswertung der untersuchten Neologismen bestätigt diese Annahme, denn von insgesamt 427 untersuchten Substantiven sind 334 Komposita.

Folgende Belege sollen das Streben nach der Informationsverdichtung in der Presse illustrieren:

Ihr Terminabsprachewunsch erbeten unter Tel. ...

<FAZ 223/1993,19>

1992 wurde auf dem Münchener Gipfel ein

24-Milliarden-Dollar-Paket für Rußland geschnürt ...

<SüdZ 222/1993,33>

Neben dem Nacht, Abschied, Tod-Themenkreis kann sich der Interessierte kleinere Zyklen ... zusammenstellen.

<SüdZ 222/1993,18>

Immerhin gelang es ... den DTB-Funktionären, ... das

Mutter-Tochter-Krankengymnastinnen-Duo ... fernzuhalten.

$<$ FAZ 223/1993,24>

Dem reichlich abstrakten Essay wäre folgende sehr reale Auswirkung der Shopping-Mall-Manie hinzuzufügen ...

$<$ Z 39/1993,88>

Dort erstreckt sich das deutsche Vorzeigpanorama, die

O-wie-schön-Landschaft aller Weintouristen ...

$<$ Z 39/1993,34>

... eben virtuoses Entertainment wie es die Europäer auch dann nicht zustandebringen werden, wenn die Franzosen es schaffen, der EG ... auch ihre Schaut nur beim Europäer-Politik aufzuzwingen.

$<$ SüdZ 222/1993,18>; 
ebenso auch Null-Toleranz-Politik, Null-Toleranz-Strategie, Rund-um-die-Uhr-Betreuung, Feine-Leute-Sport, 5-Stern-Design, 1,99-Meter-Mann, 120-EpisodenSerie, Fünf-Prozent-Grenze, Bild-Laut-Sprache, Kosten-Nutzen-Rechnungen u.a.m.

Kompositionsglieder können auch Wortgruppen und Sätze sein; es geht um die Univerbierung, um das Ergebnis eines "Zusammenwachsens mehrgliedriger syntaktischer Konstruktionen zu einem Wort" (BUßMANN 1990, 819). BRAUN (1993) stellt fest, daß die Univerbierung in der deutschen Gegenwartssprache stark zunimmt und versteht sie als "Haupttendenz im Bereich der deutschen Wortbildung" (168). Bei der Univerbierung von Wortgruppen geht es eigentlich um die substantivische Konversion; eine Wortgruppe wird zum Substantiv, wie etwa Rechtssein, Schulterklopfen, In-den-Arm-Nehmen, Nichteinverstandener, Gewordensein.

Die zitierten Belege weisen also eine Art syntaktischer Vereinfachung und zugleich eine Informationsverdichtung im substantivischen Bereich nach.

Die stark ausgeprägte Kompositionsaktivität des Substantivs zeigt sich auch in der Neigung zur Reihenbildung. Bestimmte Substantive treten reihenhaft als Erst- oder Zweitglieder in die Komposition ein, z.B. UNO-, US-, EG-, PR-, Auto-, Recycling-, Ost-, Schnupper- als Erstglied bzw. -recycling, -happening, -desaster, -generation als Zweitglied.

Für die extrem hohe Frequenz der neuen Substantive spricht auch die Tatsache, daß in den Wortschatz der deutschen Tages- und Wochenpresse fach- und sondersprachliche Lexik eindringt. Das gilt besonders für diejenigen Fachbereiche, die in den Tageszeitungen eigene Rubriken haben, z.B. Sport, Wirtschaft, Politik, Kultur, oder für diejenigen, die durch die Tagespresse popularisiert werden. So wird z.B. über EDV, Tourismus, Ökologie oder Verkehr oft geschrieben, da diese Fachbereiche von allgemeiner Bedeutung sind, denn sie betreffen immer mehr das alltägliche Leben. Die Fach- und Sondersprachen weisen meist einen hohen Anteil an Substantiven auf. So können folgende substantivische Neologismen zur fach- und sondersprachlichen Lexik gezählt werden:

Der Erfolg ist, daß Phoenix die höchste Recyclingquote aller amerikanischen Städte hat ...

$<$ FAZ 223/1993,10>

Unentwegt ist von Nutzen-Kosten-Analysen ... die Rede ...

<SüdZ 222/1993,3>

Im sechsten Spiel nahm der Deutsche seinem Kontrahenten den Aufschlag ab, ... und rettete den Vorsprung zum 6:3-Satzgewinn ...

<SüdZ 222/1993,46>

Bei Auslands-Überweisungen kassieren die Banken trotz EG-Binnenmarkt kräftig ab.

<Z 39/1993,39>; 
ebenso Abfallsystem, G7-Treffen, Mobilfunk, Burn-out-Syndrom, Statement, Deal, Desktop-System, Laptop, code-sharing usw.

Die letzten Wortbeispiele sind Entlehnungen aus dem Angloamerikanischen. Es ist evident, daß die dominierende Rolle des Substantivs im untersuchten Korpus auch durch Entlehnungsprozesse beeinflußt wird. Nach SOMMERFELDT $(1988,159)$ sind eben Substantive die am häufigsten vertretene Wortart im Prozeß der Entlehnung. Dafür sprechen auch die Ergebnisse der vorliegenden Untersuchung, denn die meisten Entlehnungen sind Substantive.

Es ist schließlich festzustellen, daß für die Textgestaltung - und damit auch für die Wahl der Substantive - die intentionale Komponente von großer Bedeutung ist. Die jeweilige Intentionalität eines Textes wird unter anderem auch durch die Auswahl bestimmter lexikalischer Ausdrucksmöglichkeiten ersichtlich. Die Intention mancher Pressetexte besteht nämlich nicht nur in der Faktendarstellung oder Tatsachenmitteilung, sondern auch darin, beim Leser Aufmerksamkeit zu erregen und Interesse zu erzeugen. Dadurch sollen die Voraussetzungen der Kommunikation geschaffen oder verbessert werden, mit dem Leser soll ein engerer Kontakt hergestellt werden. LÜGER $(1983,61)$ spricht in diesem Zusammenhang über die "aufmerksamkeitserregende sprachliche Präsentation", die aber auch unter dem textsortenspezifischen Aspekt zu beobachten ist. Der häufige Gebrauch von meist okkasionellen substantivischen Komposita, oft Entlehnungen (vor allem aus dem Angloamerikanischen), und zwar auch in den Fällen, wo die einheimischen Wörter geläufiger wären und außerdem noch verbale Ausdrucksmöglichkeiten bestehen, spricht für die gezielte Auswahl solcher Lexik, denn sie soll beim Leser Aufmerksamkeit erregen. Von der Neuheit der okkasionellen substantivischen Neologismen geht offensichtlich ein Reiz aus.

Einige Kontextbeispiele:

Seit einigen Jahren unternimmt es die historische Semantik,

Bedeutungskarrieren aufzuzeichnen ...

$<$ Z 39/1993,79>

Der Film ist ein zwei Stunden langer Armlehnen-Kraller,

Hochspannungs-Amüsement der Oscar-Klasse.

<SüdZ 222/1993,18>

Schnupper-Mitgliedschaft in der CDU Schleswig-Holsteins stößt auf Bedenken.

$<$ SüdZ 222/1993,5>

... und die Beinahe-Gleichsetzung von Ausdruckstanz und zeitgenössischem Tanztheater ... lehnten sie ... ab ...

$<$ FAZ 223/1993,30> 
Und die Athleten aus aller Welt können sicher sein, von den Sydneysidern ... gefeiert zu werden.

$<$ FAZ 223/1993,26>.

Es kann weiter angenommen werden, daß das Interesse an der Lexik nicht nur dann wächst, wenn diese neu oder auffällig wirkt, sondern auch dann, wenn die komplexen substantivischen Neubildungen Verständnisschwierigkeiten bereiten. Mit dem Streben nach konzentrierter Information steigt auch die Schwierigkeit und Vagheit des Wortschatzes. Bei einer Reihe von Komposita sind nämlich mehrere interne Beziehungen und daher auch mehrere Bedeutungen möglich, z.B.Autotourist, Konflikt-Präsident, Konsens-Präsident, Italo-Sportler, Haupstadt-Pessimismus, wobei Autotourist entweder Tourist mit einem Auto oder Tourist in einem Auto sein kann; KonfliktPräsident und Konsens-Präsident sind entweder durch Konflikt / Konsens gewählte Präsidenten oder zum Konflikt / Konsens bereite Präsidenten; Italo-Sportler steht für einen italienischen Kleinwagen und nicht etwa für einen italienischen Sportler; Hauptstadt-Pessimismus bedeutet im untersuchten Korpus Pessimismus gegenüber Berlin als Haupstadt und nicht Pessimismus (in) einer Hauptstadt, was sonst auch möglich wäre.

Manchmal ist die aktuelle Bedeutung auch durch den Kontext schwer zu erschließen:

Nach Maastricht-Debatte, Währungsdebakel und

Bosnien-Konflikt würde die EG eine solche Belastung kaum aushalten. $<$ Z 39/1993,29>

Ein Präsidentenkandidat oder gar ein Kandidaten-Kandidat ... sollte sich schon politisch kenntlich machen dürfen.

$<$ FAZ 223/1993,10>.

Es ist nicht ersichtlich, ob es sich um einen Konflikt in oder über Bosnien handelt und ob mit Kandidaten-Kandidat ein Kandidat unter oder zum Kandidaten gemeint ist.

Die Auswahl solcher Lexik kann auch damit begründet sein, daß dem Leser einer Tageszeitung bestimmtes Vorwissen unterstellt wird: Wegen der periodischen Erscheinungsweise der Zeitungen und wegen der Tatsache, daß sie vorwiegend als Abonnementzeitungen verkauft werden, was bedeutet, daß das Publikum eine bestimmte Tageszeitung regelmäßig liest.

Zusammenfassend läßt sich feststellen, daß Substantive mit $93,6 \%$ aller Wortbeispiele die meist vertretene Wortart in der untersuchten Neologismensammlung sind. Die statistisch festgestellte Dominanz der Substantive verifiziert für die Presse charakteristische Tendenz zum Nominalstil. Die substantivischen Neologismen werden aus Gründen der gestrebten Informationsverdichtung bevorzugt oder sie gehören der fachbzw. sondersprachlichen Lexik an. Nicht selten werden sie aber gebildet und gebraucht, um beim Leser Aufmerksamkeit zu erwecken. 


\section{NEOLOGISMEN HINSICHTLICH IHRER HERKUNFT}

Entlehnungsprozesse sind für die Lexik einer Sprache besonders bedeutsame Mittel ihrer Veränderung und Erweiterung. Durch zahlreiche Sprachkontakte werden die Sprachen wechselseitig beeinflußt, was vor allem auf der lexikalischen Sprachebene zu beobachten ist. Durch die kulturelle, wirtschaftliche, soziale und wissenschaftliche Zusammenarbeit werden die sprachlichen Interferenzen und damit auch die Entlehnungsprozesse gefördert und gefordert, denn es kommt nicht selten vor, daß die lexikalischen Entlehnungen gleichzeitig mit der "entlehnten Sache" realisiert werden. Der steigende Benennungsbedarf, die neuen kommunikativen Bedürfnisse, auch das Streben nach Verdeutlichung, Differenzierung, Präzisierung und Sprachökonomie begründen und begünstigen die lexikalischen Entlehnungsprozesse.

SCHIPPAN $(1992,262)$ sieht jedenfalls auch in der Mode eine besonders starke Triebkraft der Entlehnungen; so wurde das Englische (vor allem das Amerikanische) nach 1945 zur Modesprache, was aber auch für einen (immer stärkeren) wirtschaftlichen und politischen Einfluß der USA spricht. Der Einfluß des Englischen auf das Deutsche dominiert bereits seit dem 19. Jahrhundert; zuerst des Britischen, besonders nach 1945 aber des Amerikanischen. LÜGER zählt das auffällige Eindringen von Angloamerikanismen sogar zu den Haupttendenzen im lexikalischen Bereich der deutschen Pressesprache. ${ }^{4}$ Die Ergebnisse der vorliegenden Untersuchung verifizieren diese Tendenz, denn außer Bezeichnungsexotismen (s.u.) und vereinzelten Entlehnungen aus dem Französichen und Spanischen (Amüsement, Amigo, Millionario) stammen alle übrigen entlehnten Neologismen aus dem Angloamerikanischen.

Weitere Ursachen für die stark ausgeprägten Entlehnungsprozesse liegen auch darin, daß Entlehnungen nicht selten als Prestigewörter empfunden werden. Sie gelten oft als ausdrucksstärkere lexikalische Lösungen, mit denen das Lokalkolorit erzeugt werden kann.

\subsection{ARTEN UND FORMEN DER ENTLEHNUNG}

Die entlehnten Wörter "werden üblicherweise Fremdwörter genannt, obgleich sie zu einem großen Teil gar keine fremden, sondern durchaus altbekannte, gebräuchliche und nötige Wörter innerhalb der deutschen Sprache sind" (DUDEN Fremdwörterbuch 1974, 16). Es wird in der Literatur aber oft auch zwischen Fremdwort und Lehnwort unterschieden. ${ }^{5}$ Ein Fremdwort ist danach ein aus einer fremden Sprache übernomme- 
nes Wort, das sich (noch) nicht an das Sprachsystem der aufnehmenden Sprache angepaßt hat (phonetisch, orthographisch, morphematisch). Ein Lehnwort dagegen ist ein fremdes Wort, das dem Sprachsystem der "neuen" Sprache völlig angepaßt ist und in dieses System integriert ist. Es wird damit nicht mehr als fremd empfunden.

Eine solche terminologische Abgrenzung erfolgt nach dem Assimilationsgrad eines entlehnten Wortes. Die meisten entlehnten Wörter stehen aber zwischen diesen zwei Extremwerten (vgl. SCHIPPAN 1992, 264); deswegen ist die Abgrenzung äußerst schwierig und auch nicht immer möglich, weil die Übergänge fließend sind.

Differenzierungskriterien für die Abgrenzung der Fremdwörter von den Lehnwörtern (Lautung, Akzentuierung, Orthographie, morphematische Struktur, Einnahme in die Wortbildungsparadigmen, Geläufigkeit) ${ }^{6}$ scheinen nicht fest und auch nicht immer nachweisbar zu sein. Vor allem kommt das beim untersuchten Wortmaterial zum Ausdruck. Bereits die Lautung kann bei den geschriebenen Texten oft nicht geprüft werden, da die neuen Wörter in den Wörterbüchern (auch in den Fremdwörterbüchern) selten kodifiziert sind. Oft treten bei den entlehnten Wörtern orthographische Varianten auf : Ein Zeichen dafür, daß Assimilation ein andauernder, dynamischer Prozeß ist, wobei starre Grenzen nicht festzulegen sind. Die morphematische Struktur und die grammatisch-paradigmatische Einordnung der Neologismen in die Wortbildung lassen sich beobachten, problematischer ist aber die Festellung ihrer Geläufigkeit. Da der Begriff Neologismus hier breit verstanden wird, ist der größte Teil von Neologismen im Sprachgebrauch (noch) nicht gefestigt.

Weiter kann der Ausdruck Fremdwort auch mit sprachkritischen, sprachpflegerischen, puristischen, oft auch wertenden, subjektiven und moralischen Konnotationen beladen werden.

Aus den genannten Gründen wird auf die Trennung Fremdwort : Lehnwort verzichtet und nur über das Lehnwort bzw. über das entlehnte Wort gesprochen. Dieses wird als ein Oberbegriff für Fremd- und Lehnwort verstanden. In dieser Hinsicht wird die Meinung GREULES ${ }^{7}$ vertreten; er bezeichnet "alle lexikalischen Elemente, die aus anderen Sprachen stammen" als Lehnwörter (264). Sie sind Produkte der Entlehnungsprozesse und sind mehr oder weniger an das Deutsche angepaßt. Jedenfalls werden hier aber nur diejenigen entlehnten Neologismen behandelt, die als "fremd" empfunden werden, d.h. Lehnwörter, bei denen der Prozeß der Assimilation (noch) nicht fortgeschritten ist, die noch orthographische, morphematische oder sonstige Merkmale ihrer Herkunftssprache aufweisen und damit ihre Fremdartigkeit betonen bzw. nachweisen. SPRACHE: KLEINE ENZYKLOPÄDIE (1983, 304f.); W. FLEISCHER/I. BARZ (1992, 61). 


\subsection{ENTLEHNTE WÖRTER ALS ERSTBENENNUNGEN}

Der ständige und zunehmende Bedarf an Neubenennungen in allen Lebensbereichen spiegelt sich u.a. in den Entlehnungsprozessen wider. Mit einer neuen Sache oder mit einem neuen Sachverhalt werden auch entsprechende Benennungen entlehnt. Sie fungieren dann als Erstbennenungen, da sie in der "neuen" Sprache (noch) keine einheimischen Äquivalente haben; sie üben dadurch vorwiegend kognitive Funktion aus.

Folgende Beispiele können als Erstbenennungen verstanden werden:

... und dennoch geht die Wortschöpfung zügig weiter, schlagen die Neologen immer wieder zu.

$<$ SüdZ 222/1993,18>

... bietet das Hotel ... die Ruhe seiner 50 Zimmer und Duplex ...

$<$ FAZ 223/1993,19>

Das hat jetzt der Verkaufsdirektor ... bei der Präsentation eines neuen Zusatzangebots, des McTrain, in Frankfurt versichert.

$<$ FAZ 223/1993,14>;

ebenso code-sharing, Curling, Curlerin.

Zur Kategorie der Erstbenennungen werden auch Bezeichnungsexotismen gezählt. Das sind Entlehnungen, die für die Herkunfssprache bzw. für das Herkunftsland charakteristische Gegebenheiten oder Einrichtungen bezeichnen; damit bleiben sie auf einen Begriff der fremdsprachigen Umwelt beschränkt (vgl. SCHIPPAN 1992, 266).

... so hätten sie im Sejm eine klare absolute Mehrheit.

$<$ SüdZ 222/1993,7>

Die Volksdeputierten tagen.

<FAZ 223/1993,1>

Die deutsche Niederlage bei der Abstimmung in der Knesset ... hat die LikudOpposition in eine Krise gestürzt ...

$<$ FAZ 223/1993,6>

\subsection{ENTLEHNTE WÖRTER ALS ZWEITBENENNUNGEN}

Neologismen, die als Zweitbennenungen fungieren, werden als Varianten zur einheimischen Lexik verstanden. Sie werden entlehnt, weil sie als pragmatisch günstiger gelten (vgl. SCHIPPAN 1992, 246). Die Bestrebungen nach Bedeutungspräzisierung und/oder -differenzierung und nach Sprachökonomie begründen den Gebrauch solcher 
Entlehnungen. SOWINSKI $(1991,124)$ spricht in diesem Zusammenhang über die "intentionale Funktion der Fremdwörter".

Entlehnungen werden im Vergleich zu den einheimischen Entsprechungen oft als ausdrucksstärker empfunden. Sie werden absichtlich in Konkurrenz mit den entsprechenden einheimischen Wörtern gebraucht, um bestimmte Konnotationen beim Rezipienten zu erwecken. Folgende Beispiele entstammen den Texten, die über ihr Herkunftsland sprechen. Sie sollen daher ein bestimmtes Lokalkolorit erzeugen, etwa eine amerikanische Athmosphäre schaffen:

Diese Membership sagt nichts über die Qualität des Programms aus ...

$<$ FAZ 223/1993,43>

Der Skyscraper fungiert wie ein Wegweiser.

$<$ SüdZ 222/199,29>.

Membership bezieht sich auf das amerikanische MBA-Studium und Skyscraper steht für Empire State Building; sie konkurrieren demnach intendiert mit den entsprechenden und außerdem üblichen Wörtern Mitgliedschaft und Wolkenkratzer.

Folgende Beispiele illustrieren die südafrikanischen bzw. australischen Verhältnisse:

Immerhin kommt es in den Townships ... zu Verbrüderungen zwischen verfeindeten Gruppen ...

<SüdZ 222/1993,I>

Das sind die neuen Homelands für die wenigen ... Leute, die abends noch ausgehen.

$<$ SüdZ 222/1993,I>

So eine freudige Sache war der Trip nach Sydney nicht immer.

$<$ SüdZ 222/1993,4>.

Eine Bedeutungspräzisierung und -differenzierung liegt dem Gebrauch von folgenden Entlehnungen zugrunde:

Dann werden ... gelochte Putts zum Stoff, aus dem Legenden für spätere Generationen gestrickt werden.

<SüdZ 222/1993,46>

Der Brite ... näselte in feinstem Queens-Englisch ...

$<$ FAZ 223/1993,23>.

Putt steht für Schlag bei Golf und Queen assoziiert die englische Königin; die deutschen Entsprechungen Schlag und Königin haben aber eine breitere Semantik.

Dem Gebrauch von entlehnten Wörtern werden oft Prestigewerte zugeschrieben (vgl. BUBMANN 1990, 253). SANDIG (1986, 258f.) spricht iiber die lexikalischen Prestige-Signale und betont dabei den Einfluß des Angloamerikanischen auf das Deut- 
sche. Die Angloamerikanismen werden als Prestigewörter gebraucht, obwohl es im Deutschen auch einheimische usuelle Benennungen für die gleichen Denotate gibt:

... Otto, der Entertainer wird ... eine eigene RTL-Live-Show haben.

<SüdZ 222/1993,29>

In welchem der vielen großen Londoner Parks ... kann der britische Fan ... seinem lebenslangen Open-air-Hobby nachgehen?

$<$ SüdZ 222/1993,VI>

Erholung vom frommen Sightseeing gewährt der Biergarten ...

$<$ Z 39/1993,85>

Beide Seiten sind happy.

$<$ Z 39/1993,22>;

ebenso auch Statement, recyceln, Entertainment, managing director, paper, Notebook, zahlreiche Bildungen mit Recycling-, PR-. Es ist nicht anzunehmen, daß die genannten Entlehnungen einen breiteren Bedeutungsumfang als die einheimischen Entsprechungen hätten.

Die sprachökonomischen Gründe begünstigen den Gebrauch von Entlehnungen in folgenden Beispielen, wo die einheimischen Entsprechungen bzw. Paraphrasen unter dem sprachökonomischen Aspekt ungünstiger wären:

Downtownfans schwärmen von Chicago ...

$<$ Z 39/1993,73>

... rollt mit dem TV-Start eine elefantöse Merchandising-Welle an.

<SüdZ 222/1993,29>

Ist die Rechnerei vorbei, werden nacheinander drei Disketten in den Laptop geschoben.

$<$ Z 39/1993,49>.

\subsection{ENTLEHNTE WÖRTER IM FACHWORTSCHATZ}

Die fachsprachliche Lexik wird durch Entlehnungsprozesse besonders geprägt. Die Fachausdrücke dringen aber auch in die Pressesprache, wo sie im Rahmen eines Fachbereiches als Termini oder Jargonismen fungieren.

In der untersuchten Neologismensammlung fungieren etwa folgende Entlehnungen als Termini im Bereich der Wirtschaft, Literaturwissenschaft oder des Films:

... kündigte die Fuji Bank Ltd. an, ihre kurzfristige Prime rate ... zu ermäßigen.

$<$ SüdZ 222/1993,38> 
... im Fischer Taschenbuch Verlag der Band Die Nacht in den Wäldern mit Short Stories der Amerikanerin Djuna Barnes.

<SüdZ 222/1993,IV>

An Affair to Remember ist ein Remake.

<SüdZ 222/1993,29>

Die Mischung der 13 ausgewählten Filme geht quer durch das Angebot: Krimi, Komödie, Trickfilm, Action ...

$<$ SüdZ 222/1993,29>.

Als Jargonismen sind folgende Wortbeispiele aus dem Bereich der EDV zu klassifizieren:

Die Klage der Sicherheitsfachleute dagegen lautet, daß die Codemakers den Codebreakern derzeit um Längen voraus seien.

$<Z$ 39/1993,49>.

Der EDV-Wortschatz ist durch das Angloamerikanische in höchstem Maße geprägt (vgl. KALZ 1988, 38f.). Die nächsten Beispiele gehören wegen starker Popularität des Themas bereits dem Allgemeinwortschatz an: Laptop-Computer, Computerpower, PC.

Die obigen Wörter können zu den in den Fachwortschätzen oft vorkommenden Internationalismen klassifiziert werden. Das sind Wörter, die der jeweiligen aufnehmenden Sprache morphematisch, lautlich und orthographisch angepaßt und in der gleichen Bedeutung international verbreitet sind. Über die Internationalismen berichtet ausführlich BRAUN ${ }^{8}$ und bezeichnet sie als "sprachliche Gemeinsamkeiten in Wortschätzen verschiedener Sprachen", die die Tendenz der lexikalischen Internationalisierung verifizieren (194).

Es wurde festgestellt, daß die Internationalismen durch die internationale Zusammenarbeit gefördert werden (vgl. SCHIPPAN 1992, 265); es ist aber auch ersichtlich, daß diese Zusammenarbeit sprachlich überwiegend durch das Angloamerikanische bestimmt bzw. beeinflußt wird.

\subsection{ASSIMILATIONS- UND INTEGRATIONSGRAD DER ENTLEHINTEN WÖRTER}

Im Prozeß der Entlehnung werden die entlehnten Wörter der "neuen" Sprache mehr oder weniger angepaßt, damit sie als lexikalische Einheiten funktionieren können. Je höher ist der Grad der Assimilation, desto früher wird das Wort in die "neue" Sprache 190-207. 
integriert. Es kann angenommen werden, daß für Neologismen (und noch besonders für Okkasionalismen) der Assimilationsgrad von großer Bedeutung ist, denn er begünstigt ihre Akzeptabilität, ihre Angemessenheit für die Integration in die entlehnende Sprache; er trägt zu ihrer Gebräuchlichkeit bzw. zu ihrem Bekanntheitsgrad wesentlich bei.

Die Anpassung eines entlehnten Wortes wird beobachtet an folgenden Kategorien: Lautung, Orthographie, morphematische Struktur und Einordnung in die Wortbildungsparadigmen der "neuen" Sprache.

\subsubsection{Lautung als Assimilationskriterium}

Unter Lautung werden die Lautstruktur und die Art des Aussprechens eines entlehnten Wortes verstanden. SCHIPPAN $(1992,265)$ stellt fest, daß die Lautung gleich zu Beginn der Entlehnungsprozesse dem deutschen Sprachsystem angepaßt wird. Es scheint aber, daß das (zumindest für die Entlehnungen aus dem Angloamerikanischen, die aus stilistischen Gründen intendiert entlehnt und okkasionell gebraucht werden) nicht unbedingt notwendig ist. Die folgenden Wortbeispiele erweisen eine unveränderte lautliche Struktur und es wird angenommen, daß sie auch ihrer Herkunftssprache entsprechend ausgesprochen werden:

Der Skyscraper fungiert wie ein Wegweiser ...

<SüdZ 222/1993,29>

Zugang zum amerikanischen Markt durch code-sharing gesichert.

<SüdZ 222/1993,33>

... und die Stärke des Road-Movie ... liegt in der überraschenden Photographie ...

<SüdZ 222/1993,29>

Ich bin ... kein Illustrator für einen Film, der zu neunzig Prozent aus action besteht ...

<FAZ 223/1993,VI>

... ich verachtete sogar den Rummel, der mit ... Small Talk einherging ... <SüdZ 222/1993,IIl>;

ebenso Subway, Teamcaptain, Putt, Curling, rerun, Codemaker, Codebreaker, Township, Homeland, Reihenbildungen mit Recycling- u.a.m.

Eigentlich ist für die Untersuchung der Neologismen in geschriebenen Texten die Aussprache als Assimilationskriterium weniger angebracht, da sie oft nicht geprüft werden kann, denn die Neologismen sind in den gängigen Wörterbüchern selten bzw. überhaupt nicht kodifiziert.

Es kann angenommen werden, daß die folgenden Entlehnungen in der Aussprache an das Deutsche stärker angepaßt sind; sie werden auch öfter gebraucht, und außerdem sind Aussprachevarianten möglich: 
Ist die Rechnerei vorbei, werden ... die Disketten in den Laptop geschoben. $<$ Z 39/1993,39>

... welche Software wohl den Anforderungen der nächsten PC-Generation genügen würde.

$<$ Z 39/1993,18>;

ähnlich auch Reihenbildungen mit PR- als Erstglied: PR-Berater, PR-Mann, PRWelt.

In der Lautung völlig assimiliert scheinen folgende Beispiele zu sein:

Die UNPROFOR teilte mit, zwei britische Soldaten seien ... verwundet worden ...

<SüdZ 222/1993,10>

... und die Basketballer müssen schon wieder in der Bundesliga ran ...

$<$ SüdZ 222/1993,47>;

weiter Reihenbildungen mit UNO- als Erstglied: UNO-Beitritt, UNO-Flüchtlingshilfswerk, UNO-Hochkommissariat, UNO-Kontingent u.a.m.

\subsubsection{Orthographie als Assimilationskriterium}

Orthographisch zeigt sich die Assimilation der entlehnten Wörter vor allem in der Großschreibung der entlehnten Substantive. Der überwiegende Anteil solcher Substantive sind Angloamerikanismen, z.B.: Event, Crew, Swimmingpool, Teamcaptain, Trip, Township, Care-Paket, Entertainment, Gearing, Highway u.a.m.

Es finden sich aber auch Wörter, die ihre (englische) Orthographie völlig aufbewahrt haben. SEIFERT $(1990,323)$ grenzt sie von den übrigen Entlehnungen ab und nennt sie Zitate aus einer anderen Sprache, zitierte fremde Wörter.

Einige Kontextbeispiele:

Denn diese hat sich ... von einem generationenübergreifenden rerun ... zu einem Objekt fast kultischer Verehrung entwickelt.

<SüdZ 222/1993,18>

Dieser wird dann die Weiterbeförderung der code-sharing-Flüge der Lufthansa ... übernehmen.

$<$ SïdZ 222/1993,33>

Vom gentlemens' sport Golf war ... wenig zu spüren.

$<$ SüdZ 222/1993,46>;

ebenso managing director, paper, cinephil.

Die orthographischen Varianten sprechen dafür, daß die Angloamerikanismen auch bewußt intentionell (um etwa Aufmerksamkeit zu erwecken) in englischer Schreibweise 
verwendet werden (Road-Movie oder road movie); sie können aber auch einen differenzierten Bekanntheitsgrad nachweisen.

Die unterschiedliche Orthographie stimmt wie folgt mit einer Bedeutungsdifferenzierung überein; action steht für spannende Handlung und Action für Actionfilm:

Ich bin ... kein Illustrator für einen Film, der zu neunzig Prozent aus action besteht ...

$<$ FAZ 223/1993,VI>

Die Mischung der 13 ausgewählten Filme geht quer durch das Angebot: Krimi, Komödie, Trickfilm, Action ...

$<$ SüdZ 222/1993,29>.

\subsubsection{Morphematische Struktur als Assimilationskriterium}

Die assimilierte morphematische Struktur der Entlehnungen bedeutet ihre Inkorporierung in das grammatische System der entlehnenden Sprache. Die entlehnten Wörter erhalten dabei die Merkmale der grammatischen Kategorien für die jeweilige Wortart, der sie angehören. Substantive bekommen für das Deutsche charakteristische Genus-, Kasus- und Pluralmerkmale, Verben erhalten Infinitiv- und Konjugationsformen, und Adjektive werden oft als Attribute in Kongruenz mit dem entsprechenden Substantiv gebraucht.

Jedem Substantiv wird das Genus zugewiesen. Die Genusmerkmale können dem Genus der Herkunftssprache entsprechen, sie können sich nach dem natürlichen Geschlecht richten oder sie stehen in Analogie mit dem Genus semantisch benachbarter deutscher Wörter (vgl. SCHIPPAN 1992, 265).

Folgende Entlehnungen weisen auch im Deutschen das Genus ihrer Herkunftssprache, d.h. des Englischen nach:

"Alles interessante Perspektiven", faßt Dr. P. das

Brainstorming zusammen.

$<$ Z 39/1993,44>

Mercedes könnte folglich auf hochbewährtes Knowhow

zurïckgreifen ...

<SüdZ 222/1993,46>

Das Programm ... beinhaltet ein viermaliges Gearing Ihres Kapitals.

$<$ FAZ 223/1993,10>.

Dem natürlichen Geschlecht entsprechend sind folgende Substantive Maskulina:

1947 ließ der britische Teamcaptain ... die Schläger ... kontrolieren ... $<$ FAZ 223/1993,23>

... Otto, der Entertainer wird ... eine eigene RTL-Live-Show haben.

$<$ SüdZ 222/1993,29>. 
Bei den vielen untersuchten Substantiven, die aus dem Englischen stammen, erfolgt die Genuszuweisung analog mit den semantisch identischen oder ähnlichen einheimischen Wörtern, z.B.: die Membership (die Mitgliedschaft), die Subway (die UBahn), die Crew (die Mannschaft), die Prime rate (die Rate/Zinsrate), die MedienShow (die Schau), die Computerpower (die Macht), die Business Class (die Klasse), der Trip (der Ausflug), der PR-Deal (der Handel), der Pop-Shop (der Laden), der Laptop (der Computer), der Skyscraper (der Wolkenkratzer), die UPROFOR (die Organisation/Institution UNPROFOR, wobei das Wort Organisation bzw. Institution nicht ein Bestandteil des Initialwortes UNPROFOR ist (UNPROFOR=United Nations Protection Forces).

Die entlehnten Substantive bekommen auch die für das deutsche Deklinationssystem üblichen Pluralmerkmale. Bei den meisten untersuchten Beispielen ist das ein sPlural:

Downtawnfans schwärmen von Chicago ...

$<$ Z 39/1993,73>

Und weiter ging's mit verschiedenen Events.

$<$ SüdZ 222/1993,II>

Die Flugtickets ... werden aus eigener Tasche bezahlt.

<SüdZ 222/1993,54>;

auch Codemakers, Matches, Malls, Putts, Skins, managing directors, Short Stories, Homelands, Townships.

Auffällig wirkt die Bildung einer s-Pluralform mit dem zusätzlichen Apostroph, was für das Deutsche nicht üblich ist:

Escom werde künftig ... in der Lage sein, PC's ... anzubieten.

$<$ SüdZ 222/1993,37>.

Einen höheren Assimilationsgrad an das Deutsche zeigen zwei folgende Null-Pluralformen, die auf -er auslauten; solche Substantive weisen im Deutschen einen NullPlural auf (im Dativ -n):

Früher müßten die Topmanager nur mir berichten ...

$<$ FAZ 223/1993,12>

Weber stellte fest, daß damit ... für die Lufthansa die Chance bestehe, in einem Wettbewerb mit den US-Carriern zu treten ...

$<$ SüdZ 222/1993,33>.

Die meisten Maskulina und Neutra erhalten im Genitiv Singular die s-Endung; so auch im folgenden Beispiel:

Dafür spricht ... die im Fall eines Crashs gezielt verformbare ... RahmenGrundstruktur.

$<$ SüdZ 222/1993,59>. 
Der Genitiv steht auch ohne eine explizite Bezeichnung. Bei den Eigennamen mit dem Artikel ist das üblich (des McTrain); bei des Road-Movie ist das ein Zeichen für eine (noch nicht realisierte) Anpassung an das deutsche Deklinationssystem:

Das hat ... der Verkaufsdirektor ... bei der Präsentation eines neuen Zusatzangebots, des McTrain, in Frankfurt versichert.

$<$ FAZ 223/1993,14>

... und die Stärke des Road-Movie ... liegt in der überraschenden Photographie ...

$<$ SüdZ 222/1993,29>.

Werden die entlehnten Adjektive als Attribut beim Substantiv gebraucht, dann werden sie meist auch dekliniert; sie passen sich dem Deklinationssystem des Deutschen an. Mit dem Substantiv kongruieren sie im Kasus, Numerus und Genus und zwar explizit mit den entsprechenden Deklinationsmerkmalen:

Die Serie zeigt nicht einen ultra-coolen urbanen Indy Jones ...

$<$ SödZ 222/1993,18>

Er ist eigentlich Theologe mit frühen cinephilen Neigungen ... $<$ FAZ 223/1993,V>.

Das entlehnte Adjektiv kann aber auch unflektiert verwendet werden, wobei es auch nicht steigerungsfähig ist:

... bis zum halb-happy Ende ...

$<$ SüdZ 222/1993,18>.

Die entlehnten Verben aus der untersuchten Neologismensammlung zeigen ihre Assimilation und Integration in das grammatische System des Deutschen dadurch, daß sie regelrecht konjugiert werden und daß sie ebenso die infiniten Formen nachweisen (recyceln, parexzellieren).

In erster Linie werden Kunststoffverpackungen werkstofflich recycelt ... $<$ FAZ 223/1993,9>

In seinem Gutmenschentum parexzeliert er als solcher Grobschmied, daß es weh tut.

$<$ Z 39/1993,77>.

\subsubsection{Einordnung in die Wortbildungsparadigmen als Assimilationskriterium}

Die entlehnten Wörter passen sich an die entlehnende Sprache auch so an, daß sie in ihre Wortbildungsparadigmen eingehen. Das spricht für ihre Assimiliation und begünstigt ihre Integration in die "neue" Sprache. 
Wenn die entlehnten Wörter mit den einheimischen Elementen in den WBK kombiniert auftreten, geht es um die Hybridisierung; die entsprechenden WBK sind hybride Bildungen.

Es scheint, daß die entlehnten Wörter ohne größere Restriktionen in die Komposition eingehen. In untershiedlichen Formen treten sie als Erst- oder Zweitglieder der Komposita auf.

Einige Beispiele der Komposita mit dem Lehnwort als Erstglied: Containerdorf, Fast-food-Kette, Party-Erfolg, speedgeladen, Babyurlaub, Boomjahr, GoodwillEuropäer, teamfähig, Care-Paket, Citylage, Crash-Verfahren, Performance-Maler, Stand-by-Arbeitnehmer, Jumborunde, Merchandising-Welle, Livezuschauer, Manager-Gatte, Reihenbildungen mit PR-, US-, UNO-, Recycling- als Erstglied u.a.m.

Komposita mit dem Lehnwort als Zweitglied: Zweimanncontainer, RüsselRowdy, Wasserrecycling, Fahrer-Airbag, halb-happy, Saurier-Look, reihenweise recycling und -happening u.a.m.

Nach den Modellen der deutschen Komposition können auch solche Komposita gebildet werden, bei denen beide UK Lehnwörter sind: Management-Engagement, Detail-Info, Basis-Service, Konflikt-Präsident, Konsens-Präsident Styling-Element, Kommunikationsdesign, US-Carrier, US-Caterer, ultra-cool, Desktop-System, Laptop-Computer u.a.m.

Entlehnungen treten auch in die Prozesse der expliziten Derivation ein. Sie werden entweder als Basis oder als Affix in den WBK gebraucht.

Der entlehnten Derivationsbasis werden heimische Suffixe angehängt: Basketballer, Curlerin, recyclebar, ökofundamentalistisch, Fundi, Fascho u.a.m.

Auch Wortkreuzungen und Konversionsprodukte enthalten Entlehnungen: Nolympics (No + Olympics), parexzellieren (par excellence).

Stark vertreten sind entlehnte Wörter in allen Arten der Kurzwortbildungsprodukte: z. B. Micro, Reps, Skin, Fax. Initialwörter erscheinen oft reihenhaft als Erstglieder in den Komposita: UN-, UNO-, UNPROFOR-, PC-, PR-.

In bezug auf die Herkunft des untersuchten Wortmaterials ist folgendes festzustellen:

Der Anteil an Entlehnungen, die beim Leser (und beim Schreiber) noch als fremd empfunden werden und die entweder als selbständige Lexikoneinheiten oder als Wortbildungselemente vorkommen, ist hoch, er beträgt etwa $43 \%$ aller Wortbeispiele, wobei das Angloamerikanische einen dominierenden Einfluß auf die deutsche Pressesprache hat. Das Ergebnis ist aber relativ zu verstehen, da die Abgrenzungskriterien für die Bestimmung der Fremdartigkeit eines entlehnten Wortes nicht fest definiert sind und nicht selten auf Subjektivität beruhen. Jedoch ist aber bemerkenswert, daß im Vergleich mit den Angaben im DUDEN Fremdwörterbuch $(1974,19 f$.) der Anteil der entlehnten Lexik in 20 Jahren um etwa $20 \%$ gestiegen ist. 
Relativ wenige Entlehnungen im untersuchten Korpus sind Erstbenennungen; vielmehr fungieren sie als Zweitbenennungen. Das bedeutet, daß sie absichtlich verwendet werden, um mit einheimischen Benennungen semantisch und stilistisch zu variieren, um ein bestimmmtes Lokalkolorit zu erzeugen, um eine Bedeutungspräzisierung und -differenzierung zu kennzeichnen, um als Prestigewörter zu gelten oder um sprachökonomische Tendenzen in der Sprache zu unterstützen. Es ist weiter festzustellen, daß einen wesentlichen Anteil an der entlehnten Lexik in der Presse auch Fachwörter haben, insbesondere in Kommunikationsbereichen Wirtschaft, EDV, Luftverkehr, Film, Sport.

Aus dem untersuchten Wortmaterial geht weiter hervor, daß die Entlehnungen an das Deutsche unterschiedlich angepaßt sind. Ihre Assimilation zeigt sich weniger in der Lautung und Orthographie (außer Großschreibung der Substantive), wohl aber stark in ihrer Annäherung an das grammatische System des Deutschen und in ihrer Einnahme in die Wortbildungsprozesse - hier scheinen die Entlehnungen stark assimiliert zu sein, was ihre Integration in das Deutsche begünstigt.

\section{NEOLOGISMEN HINSICHTLICH IHRER FUNKTIONALITÄT}

Neologismen erscheinen in Texten und Texte sind Teile der kommunikativen Prozesse. Die vielen sprachlichen und außersprachlichen Faktoren der Kommunikation bewirken wesentlich auch die Bildung und Verwendung von lexikalischen Innovationen. So stehen die Fragen über die verschiedenen Formen von Neologismen immer in Verbindung mit den Fragen über die Intentionen und Funktionen, die der Neologismenbildung und dem Neologismengebrauch zugrunde liegen. Es wäre deshalb ungenügend, sie isoliert von den vielen Kommunikationsbedingungen zu betrachten.

Unter Funktion wird eine Wirkungsweise verstanden, "die der Intention des Autors und der Rezeption des Lesers übergeordnet ist und beide verbindet" (SCHNEIDER 1993, 138). Die Funktion ist demgemäß eine intendierte, zielgerichtete, beabsichtigte Wirkung, die durch unterschiedliche sprachliche und/oder außersprachliche Mittel realisiert wird.

LÜGER (1983, 18f.) unterscheidet drei wichtige Funktionen der publizistischen Texte: Information, Meinungsbildung und Unterhaltung. ${ }^{9}$ Auf der sprachlichen Ebene entsprechen ihnen spezifische Formen der Stoffdarbietung: Er spricht über die tatsachenbetonten, meinungsbetonten und phantasiebetonten Darstellungsformen. Unter Darstellungsformen versteht er Textsorten, d.h. Textklassen, Gruppen von Texten, Sprechhandlungsschemata, die teilweise musterhaft und nach bestimmten

9 Ähnlich dazu C. GOOD (1989, 8); die Funktionen der Zeitungen sind Informieren, Kommentieren und Unterhalten. 
Strategien gestaltet werden und spezifische Vermittlungsaufgaben erfüllen. Sie sind demgemäß zielgerichtete sprachliche Äußerungen; sie beweisen immer eine Intentionalität, welche entweder in Faktendarstellung bzw. Tatsachenmitteilung oder in Stellungnahme oder Wertung besteht. Die jeweiligen intentionalen Komponenten sind für die Textgestaltung von großer Bedeutung, da sie die Textkonstruktion wesentlich beeinflussen. Diesbezüglich ist auch die Auswahl der Lexik zu einem großen Teil als Erscheinungsform von Intentionen zu interpretieren.

Es stellt sich weiter die Frage, wie die Textintentionen identifiziert werden (LÜGER 1983, 54f.). Welche sind sprachliche und außersprachliche Indizien einer bestimmten Intention? Aufgrund der unterschiedlichen Intentionen unterscheidet LÜGER fünf Textintentionstypen, die für die Presse charakteristisch sind und zu denen sich entsprechende Gruppen oder Klassen von Texten - Pressetextsorten zuordnen lassen.

1. Informationsbetonte Texte sind Texte, deren primäres Ziel Tatsachenmitteilung ist; wichtig sind Faktendarstellungen, Ereignisdarstellungen. Hierher gehören etwa folgende Textsorten: Nachricht, Meldung, Bericht, Reportage, Wetterbericht. Sie entsprechen der Grundfunktion der publizistischen Texte, der Information, welche eine lange Zeit sogar "die alleinige publizistische Aufgabe von Zeitungen" (LÜGER 1983, 66) war.

2. Meinungsbetont-persuasive Texte vermitteln Bewertungen, persönliche Stellungnahmen zu einem Sachverhalt; sie sind (explizite) Äußerungen von Meinungen, Urteilen, Kritiken, und der Leser möge sie akzeptieren. Dazu gehören die Pressetextsorten Kommentar, Leitartikel, Glosse, Kolumne, Kritik.

3. Instruierend-anweisende Texte enthalten direkte Aufforderungen in der Form von Handlungsanleitungen oder Ratgebungen, die für den Leser mehr oder weniger verbindlich sind. Das sind etwa Pflege- und Gebrauchsanleitungen, Kochrezepte, Verhaltensratgebungen, Horoskop.

4. Bizentrierte Texte sind Texte, bei denen zwei gleichwichtige Textintentionstypen vorhanden sind; etwa die Textsorte Interview (Wechsel von Fragen und Antworten).

5. Kontaktherstellende Texte - ihre Intention besteht darin, beim potentiellen Leser Aufmerksamkeit und Interesse zu erzeugen, Voraussetzungen der Kommunikation zu schaffen oder zu verbessern. LÜGER spricht in diesem Zusammenhang eher über eine intendierte Gestaltung der Titelseiten von Zeitungen bzw. über die funktionsbestimmten Wechselbeziehungen zwischen Schlagzeile und Illustration als über besondere Textsorten.

BURGER (1990, 30) problematisiert die Existenz von Textintentionen bei LÜGER, da Intention ein sprechakttheoretischer Begriff ist und ein Subjekt voraussetzt, welches Intentionen haben und realisieren kann. Diesbezüglich stellt sich die Frage, wer z.B. das Subjekt bei den Pressetextsorten Nachricht oder Meldung - die sind oft Ergebnisse von Nachrichtenagenturen - ist. BURGER verweist auf das Kriterium der 
Text-Funktion als Prinzip der Textsortenunterscheidung $(1990,232)$ und betont zwei für die Presse relevanten Textfunktionen: Informations- und Appell-Funktion. So unterscheidet er Texte mit dominierender Informationsfunktion, welche Lügers informationsbetonten Texten entsprechen und Texte mit dominierender Appellfunktion, welche mit Lügers meinungsbetont-persuasiven und instruierend-anweisenden Texten verglichen werden können. ${ }^{10}$

Die dargestellten terminologischen Unterschiede bzw. die unterschiedlichen Differenzierungskriterien von Pressetextsorten beweisen unterschiedliche Textsortenauffassungen. Für unsere Neologismenuntersuchung sind sie insofern relevant, da die untersuchten Neologismen von der Informations- und Appellfunktion der Texte geprägt sind. Oder nach Lügers Textsortendifferenzierung: Sie entstammen den informationsbetonten und/oder meinungsbetont-persuasiven Pressetextsorten und unterstiutzen damit die jeweiligen textsorteneigenen Intentionen. Diesbezüglich stellen sich folgende Fragen:

1. Werden die Neologismen absichtlich, intentionell gewählt, um unterschiedliche Textfunktionen zu unterstützen bzw. wie wird die Funktion als eine zielgerichtete Wirkung lexikalisch realisiert?

2. Unterscheiden sich die Neologismen, die in informationsbetonten Textsorten vorkommen, von denjenigen, die den Texten mit der dominierenden Appellfunktion eigen sind?

\subsection{NEOLOGISMEN IN TEXTSORTEN MIT DOMINIERENDER INFORMATIONSFUNKTION}

Die Grundfunktion der informationsbetonten Pressetextsorten ist die Übermittlung von Tatsacheninformationen. Der Leser soll sachlich, ohne Autors Einstellungen und Kommentierungen, wohl aber möglichst umfassend informiert werden.

GOOD $(1989,111 \mathrm{f}$ ) spricht in diesem Zusammenhang über die faktenbetonten Zeitungsgattungen. Das möge für die Textsorten Nachricht im Sinne von hard news (LÜGER 1983, 66f.) und Meldung gelten; die wertenden Elemente (zumindest explizit ausgedrückten) sollten hier fehlen. Bereits bei der Textsorte Nachricht (soft news), noch mehr aber bei Bericht und Reportage - diese werden ebenso zu den informationsbetonten Textsorten gezählt - geht es aber nicht nur um eine Informationsvermittlung, sondern sind auch wertende Elemente vorhanden. Sie zeigen sich etwa in lektüre-

10 Vgl. auch K. BRINKER (1988, 97f.). Er konstatiert folgende textuelle Grundfunktionen: Informationsfunktion, Appellfunktion, Obligationsfunktion, Kontaktfunktion und Deklarationsfunktion. Die Informationsfunktion ist charakteristisch für die Textsorten Nachricht, Bericht und Beschreibung; Texsorten mit appellativer Grundfunktion sind Kommentar, Gebrauchsanweisung, Werbeanzeige u.a.m. 
anregenden, ironischen oder humorvollen Formulierungen in soft news. In Berichten sind nicht selten auch kommentierende Stellungnahmen oder Hintergrundinformationen zu finden, und Reportagen als Pressetextsorten werden regelmäßig durch die Perspektive des Autors bzw. der Position betreffender Zeitung geprägt. Es kann bei den genannten Textsorten nicht mehr nur über eine Informationsvermittlung die Rede sein. Die informative Funktion bestimmter Textsorten steht demzufolge in einer engen Beziehung zur persuasiven Funktion; die beiden können nicht unproblematisch voneinander getrennt werden. Durch die Übermittlung von Tatsacheninformation sollte ein hoher Grad der Objektivität in der Presse gewährleistet werden, es ist aber gleichzeitig festzustellen, daß eine reine Objektivität generell nicht möglich ist. Bereits die Themenauswahl beruht auf Subjektivität; diese muß also nicht immer explizit ausgedrückt werden, um in einem Pressetext zu existieren.

Neologismen, die vorwiegend eine Information vermitteln sollen, zeigen oft eine komplexe lexikalische Struktur. Sie sind Komposita, an deren Stelle aber auch Simplizia mit Genitivattribut oder Präpositionalkasus verwendet werden könnten:

Ein ... Hilfskonvoi des UNO-Flüchtlingshilfswerks ist ... in Mostar eingetroffen.

$<$ SüdZ 222/1993,10>

Eine, von der Schwarzmeer-Flotte eingeleitete Rettungsaktion ... verzögerte sich ...

<SüdZ 222/1993,7>

... Ordnung, die sich ... nach der Beendigung des

Ost-West-Gegensatzes zu einem kollektiven System ... entwickelte.

<SüdZ 222/1993,8>

Doch nach dem August-Putsch kam es ... zu Spannungen.

$<$ FAZ 223/1993,2>;

ebenso September-Umfrage, Antiterror-Einheit, Rund-um-die-Uhr-Betreuung, Null-Toleranz-Politik, zahlreiche Bildungen mit UNO-, EG-, PR- als Erstglied.

Das sprachökonomische Prinzip zeigt sich auch bei den folgenden Klammerformen, wobei das mittlere Element reduziert wird:

Bundesrat verwirft Pflege-Konzept der Regierung.

<SüdZ 222/1993,2>

Bundesrat lehnt Pflegegesetz in erster Lesung ab.

$<$ FAZ 223/1993,11>

Chemiestandorte im Osten bleiben erhalten.

<FAZ 223/1993,11>.

Es wird angenommen, daß der Leser über ein Vorwissen verfügt, welches auch auf die Tatsache zurückzuführen ist, daß er eine bestimmte Tageszeitung (Abonnementzei- 
tung) regelmäßig liest. Er soll deswegen die erwähnten Wörter als Pflegeversicherungs-konzept, Pflegeversicherungsgesetz bzw. Standorte der chemischen Industrie verstehen.

Trotzdem ist manchmal eine erschwerte Dekodierung anzunehmen, obwohl das der Verständlichkeit, die aufgrund der informierenden Grundfunktion vorausgesetzt wird, widerspricht. Das möge etwa für die folgenden Wortbeispiele gelten:

Sie kommen ... für eine Ein-Prozent-Stichprobe von Gebäude und Wohnungen.

$<$ SüdZ 222/1993,5>

Regierungskoalition und SPD haben im Bundestag ihren Meinungsstreit in der Blauhelmfrage fortgesetzt.

$<$ SüdZ 222/1993,6>.

Es ist fraglich, ob der Leser darunter immer eine Umfrage, zu der ein Prozent der Bewohner einbezogen wird und eine Frage über die Teilnahme der Bundeswehr an den Blauhelm-Einsätzen versteht. Nicht selten muß für die korrekte Dekodierung ein breiterer Kontext einbezogen werden.

Ähnlich scheinen die Komposita mit geographischen Namen als Erstglied zu sein, wie etwa Somalia-Aktion, Hamburg-Wahl, Moskau-Hilfe, Hongkong-Frage. Geographische Namen fungieren entweder als lokale Bestimmungen (Aktion in Somalia, Wahl in Hamburg) oder sie sind ein Ziel (Hilfe für Moskau) bzw. ein Thema (Frage über Hongkong).

Die folgenden Beispiele erweisen sich als eine Art lexikalischer Varianz, d.h. Verwendung verschiedener Ausdrücke für gleiche Sachverhalte: Kosten-Nutzen-Kalkül, Nutzen-Kosten-Analyse, Kosten-Nutzen-Rechnung, Fünf-Prozent-Grenze, FünfProzent-Hürde. Das bestätigt die Tatsache, daß auch in den Textsorten, die primär der Informationsvermittlung dienen, die jeweilige Auswahl der Lexik den subjektiven Auswahlkriterien stärker untergeordnet ist, als das zu erwarten wäre.

\subsection{NEOLOGISMEN IN TEXTSORTEN MIT DOMINIERENDER APPELLFUNKTION}

Die Pressetextsorten Kommentar, Glosse, Leitartikel, Kolumne, Kritik u.ä. erweisen eine dominierende Rolle der Appellfunktion. Nach LÜGERS Einteilung gehören sie zu den meinungsbetont-persuasiven Textsorten. Ihre Textintention besteht darin, persönliche Einstellungen, Meinungen, Kommentierungen, die der Autor zu einer bestimmten Information hat, zu übermitteln und dadurch die Meinungen und Überzeugungen beim Leser zu beeinflussen. Im Vordergrund steht nicht die Informationsvermittlung, sondern die Äußerung von Kritik, Meinung, Urteil zu einer bestimmten In- 
formation, die der Leser wahrnehmen und möglicherweise übernehmen möge. Einige Kommunikationswissenschaftler stellen aber fest, daß der überredende Funktionsaspekt und damit die persuasive Wirkung bestimmter Pressetextsorten nicht in Meinungsveränderung liegt, sondern vorwiegend in Verstärkung bereits bestehender Meinungen. Das bedeutet, daß die Massenmedien - darunter auch die Tagespresse - bestehende Meinungen und Einstellungen beim Leser eher verstärken als verändern. ${ }^{11}$ Die Ursachen dafür liegen unter anderem auch im kommerziellen Charakter der Presse, denn Meinungsverschiedenheiten bzw. Auseinandersetzungen mit dem Leser werden vermieden. Es handelt sich um eine gewisse Anpassung an das Publikum. Schließlich hat aber auch der Leser immer eine Auswahlmöglichkeit; er kann aus unterschiedlichen Motiven seine Lektüre selbst auswählen. Die persuasiven Möglichkeiten der Presse scheinen also begrenzt zu sein.

Hinsichtlich der Appellfunktion von Pressetextssorten unterscheiden BRINKER (1988, 101f.) und BURGER (1990, 324) zwei Aspekte. Einerseits soll der Autor den Leser dazu bewegen, eine bestimmte Einstellung oder Meinung zu akzeptieren, und andererseits soll der Leser eine bestimmte Handlung vollziehen. Es geht um Meinungsbzw. Verhaltensbeeinflussung.

Die Appellfunktion bzw. die meinungsbetont-persuasive Textintention wird durch die gezielte Lexikauswahl realisiert bzw. unterstützt. Das bedeutet, daß auch die in der Tagespresse verwendeten Neologismen bestimmte Mitinformationen tragen bzw. daß sie neben den referentiellen Merkmalen auch wichtige Konnotationen aufweisen. Bei der Untersuchung der exzerpierten Neologismenbeispiele hat sich herausgestellt, daß die Neologismen folgende Textintentionen unterstützen:

- sie drücken persönliche Meinungen, Wertungen und Einstellungen des Schreibers aus;

- $\quad$ sie betonen die Relevanz der Gegebenheiten, für welche sie stehen;

- sie haben die Aufgabe, einen Sachverhalt zu verschleiern, zu verhüllen, sie üben damit eine euphemistische Funktion aus;

- sie werden gebildet und verwendet, um beim Leser das Leseinteresse zu erzeugen.

Es muß aber betont werden, daß die erwähnten Kategorien von Textintentionen eng miteinander verbunden sind und daß einem Neologismus oft mehrere Intentionen zugleich zugrunde liegen.

11 Es geht um die Feststellung des amerikanischen Kommunikationswissenschaftlers Joseph $\mathbf{T}$. Klapper. Zitiert nach: WIRKUNGEN der Massenkommunikation. Hrsg. von R. Burkart. Wien $1989,23$. 


\subsubsection{Neologismen als Ausdruck der Wertung, Einstellung, persönlichen Meinung des Schreibers}

Durch den Gebrauch bestimmter Neologismen können persönliche Einstellungen, Meinungen bzw. Bewertungen vermittelt werden. Sie sind entweder positiv oder negativ und sind oft in den Textsorten Kritik, Kommentar, Glosse zu finden.

Positive Bewertungen werden oft bildhaft mit Komposita ausgedrückt, die eine unerwartete, ungewöhnliche Kombination von Konstituenten aufweisen:

Sie ist ... Pop-Ikone.

$<$ FAZ 223/1993,V >

Zur markanten Frontpartie kommen die Leichtmetallräder im 5-Stern-Design

$\cdots$

$<$ Z 39/1993,27>

... schließlich beschrieb der Stilforscher die reine Formmusik der Fassaden und Höfe ...

$<$ FAZ 223/1993,29>.

Positive Einstellungen werden auch durch die häufige Verwendung bestimmter Wörter bzw. bestimmter Wortbildungselemente vermittelt. Reihenhaft gebildete Komposita mit Öko-, Recycling- oder Spar- mögen beim Leser positive Assoziationen erwecken; nicht selten wird für die Wörter bzw. für das Bezeichnete explizit geworben:

Auch durch Wasserrecycling ... lassen sich große Einsparungen erzielen.

$<$ Z 39/1993,43>

Wir müssen uns tatsächlich auf das Autorecycling einstellen ...

$<$ Z 39/1993,44>

Doch bedarf es mehr Zeit ... um dem recyclingbewußten Sammeleifer der Bürger entsprechende Verwertungskapazitäten zu bieten.

$<$ FAZ 223/1993,9>

Das Sparpaket der Bundesregierung komme eher zu spät als zu früh ... $<$ FAZ 223/1993,2>;

ähnlich Fahrzeug-Recycling, Recyclingverfahren, Recyclinganlage, Öko-Institut, Öko-Manager, Öko-Klo, Öko-Auto, Sparpapier, Sparkurs.

Wenn solche Neologismen häufig vorkommen, können sie zu Modewörtern bzw. Schlagwörtern werden. BRAUN (1993, 207f.) spricht über Schlagwörter, die "als Sprachmittel mit stark inhaltlicher Ausrichtung verstanden werden müssen, die nicht selten an Prozessen der politischen Meinungsbildung beteiligt sind"(207). ${ }^{12}$ Es geht of- 
fensichtlich nicht nur um die subjektive, persönliche Einstellung des Autors, sondern um die von ihm vertretende, wertende Einstellung der Gesellschaft, die auch in der Lexik Unterstützung findet.

Die Neologismen können aber auch abwertende, pejorative Konnotationen und eine damit verbundene negative Wertung mitbringen:

... der Bundeskanzler wolle ... die geplante Beute ... Kulturwilderern und jägern zum ... Teil entreißen.

<SüdZ 222/1993,17>

Die Verbände aus dem Norden warfen Büchner ein typisch bayerisches Amigo-Geschäft vor.

<SüdZ 222/1993,46>

Recht geschieht ihnen, den neuen Millionarios.

$<$ SüdZ 222/1993,48>.

Es ist ersichtlich, daß die abwertende Konnotation auch ironische Merkmale mitbringt. In den letzten zwei Beispielen beweisen das die Entlehnungen aus dem Spanischen: Sie assoziieren nämlich eine südliche, lässige, ungezwungene Athmosphäre, die mit ernsten Geschäften in einer ernsten Gesellschaft (EG) nichts zu tun haben sollte.

$\mathrm{Zu}$ einer negativen Wertung können auch Wortbildungselemente beitragen, wie z.B. die Suffixe -ei und -ling, die nicht selten auch pejorative Konnotationen aufweisen:

Fürsten, Grafen ... gelten als Inbegriff des arroganten Wessis, der sich anschickt, Ostländereien zu erobern.

$<$ SüdZ 222/1993,33>

... antwortete sie, daß man diese Lästlinge verunsichere, wenn man die Wohnung abkühlen lasse.

$<$ SüdZ 222/1993,18>.

\subsubsection{Neologismen als Ausdruck der Bedeutungsverstärkung}

Der gezielte Gebrauch bestimmter Lexik für einen Sachverhalt kann dessen Bedeutung hervorheben, verstärken. Damit verbunden werden auch positive Wertungen bzw. Exklusivität einer Gegebenheit ausgedrückt:

Im Urteil der Medien- und PR-Welt ist das ein Mangel.

<Z 39/1993,67>

Das Kunsthaus "Tacheles", fest etablierter Szenetempel, war keine fünf Minuten weit weg ...

<SüdZ 222/1993,II> 
Sie sollte ins Schwellenjahr 2000 weisen.

<SüdZ 222/1993,47>

Als sich am Montag ... die Jumborunde zusammensetzte, war allen klar, daß Frankreich bereit war, den großen Krach zu riskieren.

$<Z$ 39/1993,29>

Der Machtkampf zwischen Präsident Jelzin und seinen im Weißen Haus an der Moskwa tagenden Gegnern hat sich ... zugespitzt.

$<$ FAZ 223/1993,1>.

Es ist dabei nicht klar, ob die mit Weißes Haus genannte russische Regierung etwa mit der amerikanischen Regierung gleichgesetzt wird bzw. werden möchte oder geht es eher um eine ironische Betrachtung und Benennung der politischen Gegebenheiten in Rußland.

Die bedeutungsverstärkende Funktion haben auch solche Neologismen, die aus dem Angloamerikanischen entlehnt werden. In Konkurrenz mit den einheimischen Wörtern werden sie absichtlich gewählt, um die Gegebenheiten ihres Herkunftslandes dem Leser näherzubringen: Membership, Skyscraper, Homeland, Township u.a.m.

\subsubsection{Neologismen als Euphemismen}

Die Neologismen haben nicht selten eine Verschleierungsfunktion, sie sind Euphemismen. Aus unterschiedlichen Gründen der Rücksichtnahme mildern oder verhüllen sie einen Begriff, einen anstößigen, unangenehmen Sachverhalt, der beim Leser negative oder ablehnende Assoziationen bewirken könnte.

Es geht oft um eine gezielte Verharmlosung von Problemen. So werden Abfallbeseitigung und damit verbundene Schwierigkeiten mit dem Wort Abfallsystem gemildert; keine Handlung ist eine Nicht-Handlung, keine Farbe eine Nichtfarbe; Gegner sind Nichteinverstandene und die finanziellen Verluste werden Minus-Geschäfte genannt.

Die Presse kann mit einer gezielten Wortauswahl die erwünschten - meist positiven - Einstellungen $\mathrm{zu}$ - meist negativen - wirtschaftlichen oder politischen Gegebenheiten unterstützen:

Mit Nullrunden ... will Superminister ... die größte Wirtschaftskrise ... meistern.

<Z 39/1993,44>

Bayerns Innenminister ... nennt das Null-Toleranz-Politik.

<Z 39/1993,7>

Justiz und Polizei sind mit ihrer Null-Toleranz-Strategie auf dem richtigen Weg.

$<$ Z 39/1993,7> 
Nach Währungsdebakel ... würde die EG eine solche Belastung kaum aushalten.

$<$ Z 39/1993,29>.

Die extrem negativen Werte (Verzicht auf staatliche Geldmittel, Politik/Strategie ohne Toleranz, Mißerfolg bei der Einführung einer einheitlichen Währung) werden verhüllt. Es kann aber auch geschehen, daß ein Euphemismus bereits durch den Kontext, in dem er erscheint, durchsichtig wird; die positiven Werte, welche ein solcher Neologismus impliziert (Helferland, Geberland), können sogar im gleichen Satz explizit enthüllt werden:

... der größte Teil der Gelder fließt ins Helferland ... zurück.

<SüdZ 222/1993,33>

Die Gelder für Osteuropa stammen aus allzuvielen Töpfchen und nützen manchmal mehr den Geberländern.

$<$ SüdZ 222/1993,33>.

Eine bestimmte Verharmlosung problematischer Gegebenheiten kann auch ironische Effekte mitbringen:

Er gedachte, eine Ausruhbar für seine Mädchen einzurichten.

$<$ SüdZ 222/1993,II >

Indem sie die typischen Situationen ... auswendig lernen, sich auf den stereotypen Vorstellungstourismus ...begeben und hoffen, ... den Erwartungen ... gerecht zu werden.

$<$ FAZ 223/1993,43>

"Natürlich kann es sein, daß radikales Einsammeln ... die kleingewordenen Bestände weiter dezimiert", meint Pfeifer, aber eine Zunahme des Pilztourismus kann er nicht feststellen.

<SüdZ 222/1993,16>

Warum ... müßten junge Mitarbeiter ... sofort auf Ausbildungstour im Unternehmen gehen, trotz oder wegen der überlangen Studienzeiten?

$<$ SüdZ 222/1993,73>;

wobei mit Ausruhbar, Vorstellungstourismus, Pilztourismus und Ausbildungstour Bordell, Vorstellungsgespräche bei Arbeitgeber, Reisen, um Pilzen zu sammeln und zusätzliche Ausbildung gemeint sind.

Wenn Freizeit mit dem Wort Nichtarbeitszeit genannt wird, soll damit eine favorisierte Sehweise ausgedrückt werden. Die semantische Betonung liegt auf -arbeit-:

... daß einem Chefredakteur ... nicht genug Zeit für ein Kind bleibt, selbst wenn fast die gesamte Nichtarbeitszeit ihm gewidmet wird.

$<$ Z 39/1993,96>. 
Eine ähnliche Absicht wird etwa im folgenden Beispiel evident: Ein Einkaufszentrum wird zu einem Einkaufspark und dadurch soll auch die Verbrauchergesellschaft als problemlos verstanden werden:

Das Waldstück ... liegt ... rund 400 Meter von einem Einkaufspark ... entfernt.

$<$ FAZ 223/1993,7>.

\subsubsection{Neologismen als Leseanreiz}

Schließlich werden die Neologismen gebraucht, um beim Leser Leseinteresse zu erzeugen; sie sollen seine Aufmerksamkeit lenken. Die lexikalischen Innovationen als eine bildhafte Ausdrücksweise tragen zur Expressivität des Textes bei und werben für das Lesen.

Die Neologismen entstehen oft mit dem Übergang aus einem Kommunikationsbereich in den anderen oder Wortbildungselemente in einer Wortbildungskonstruktion entstammen unterschiedlichen Kommunikationsbereichen. Thre Kombinationen werden deswegen nicht erwartet. Oft kommt es zu metaphorischen Übertragungen, welche ungewöhnlich und daher expressiv, auffallend wirken:

Journalistenpoesie: Autodämmerung.

$<$ Z 39/1993,67>

Der Weinsee wächst ...

$<$ Z 39/1993,34>

Das eröffne deutschen Aktien noch einige Kursphantasie.

$<$ FAZ 223/1993,15>

IBM steckt in der Krise, und das liegt nicht zuletzt an zwei

Turnschuh-Unternehmern.

$<$ Z 39/1993,20>

Für jede Fenstergeometrie mit spitzen oder stumpfen Winkeln ...

<SüdZ 222/1993,11>

Neben einer Streichelwiese ermöglicht noch einmal ein "Affenland" die unmittelbare Begegnung zwischen Mensch und Tier ...

$<$ Z 39/1993,22>;

oder ähnlich: Dollarmeer, Hochhausgewitter, Kursfeuerwerk, Charakterzoo, HoloKitsch, Imbißhappening, Haushaltsbremse, Wirtschafts-Schlacht.

Die Ursachen für die besondere Auffälligkeit eines Neologismus sind auch in einem nicht notwendigen und daher nicht erwarteten Gebrauch von Entlehnungen zu suchen. Auf die Verwendung von Angloamerikanismen als Zitatwörter wurde bereits hingewiesen. Hier noch einige Beispiele: 
Und die speedgeladene Zeit wirkte wie ein Trommelfeuer für die neunziger Jahre.

$<$ SüdZ 222/1993,II>

$\mathrm{Zu}$ den Eröffnungen gab es fortan keine Gratisdrinks mehr.

$<$ SüdZ 222/1993,II>

Dem reichlich abstrakten Essay wäre folgende sehr reale Auswirkung der Shopping-Mall-Manie hinzuzufügen ...

$<$ Z 39/1993,88>.

Die Neologismen können auch wegen ihrer Aktualität auffällig wirken; desto mehr, wenn sie in unerwarteten Umgebungen verwendet werden. Folgende Beispiele erweisen die Beliebtheit der Dinosaurier im Jahr 1993 und sind ein Zeichen dafür, wie schnell die Sprache auf die außersprachliche Realität reagiert und wie stark ihre Innovationskraft ausgeprägt ist:

"Dinosautos als Zukunftsvision", sagt Dr. P.

$<$ Z 39/1993,44>

Autos im Saurier-Look!

$<$ Z 39/1993,44>

... und die Bohrmaschinen bohren, und mit jedem Meter ... rückt der Tag näher, an dem die stählernen Meeressaurier ... nicht mehr über den Belt dampfen werden.

$<$ Z 39/1993,83>.

Jedenfalls erscheinen solche Neologismen meist in den Textsorten Glosse oder etwa Kommentar, wo sie auch eine unterhaltende Funktion ausüben und wiederum ironische Konnotationen mitbringen:

"Also", fordert Dr. P., "müssen wir kein neues Auto projektieren, sondern das Stauto. Ein Stehzeug."

$\cdot<$ Z 39/1993,44>

... worauf mein Manager-Gatte mir natürlich mangelnde Flexibilität vorwirft ...

$<$ SüdZ 222/1993,73>

Deutsche Forscher, die etwas zu sagen haben, publizieren ihr paper am liebsten gleich in dieser Sprache.

$<$ Z 39/1993,53>.

Die Neologismen unterstützen den zugespitzten, polemischen Stil in den genannten Textsorten; daher wirken sie eher ironisch und unterhaltend als überzeugen wollend. Es steht aber auch fest, daß der Leser für die richtige Dekodierung über ein bestimmtes Vorwissen zum betreffenden Thema verfügen muß. Er bestimmt demnach ihre Wirksamkeit wesentlich mit. 
Das Leseinteresse kann schließlich auch mit Verwendung solcher Neologismen gesteigert werden, die sich an den Leser gewissermaßen anpassen. Der Schreiber will einen näheren Kontakt zum Leser herstellen oder einen bereits bestehenden Kontakt verbessern. Absichtlich verwendet er umgangssprachliche Elemente, wie z.B. folgende Kurzwörter:

... wir sind keine Fundis ...

$<$ Z 39/1993,4>

... warum eigentlich nicht "Bundis in Buchenwald"?

$<$ Z 39/1993,77>

Wenn sie aber, wie Hochhuth in seinem merkwürdigen Drama von den "Wessis in Weimar" ... von der Wirklichkeit abschreiben ..., dann haben sie verloren ...

$<$ Z 39/1993,77>;

ebenso Fascho, Juso-Zeit, Reps, Normalo.

In bezug auf die gestellten Fragen über die Funktionalität der Neologismen ist festzustellen, daß die Neologismen absichtlich, intentionell gebildet und gewählt werden, um unterschiedliche Textfunktionen der Pressetextsorten zu unterstiutzen. Die untersuchten Neologismen sind grundsätzlich von zwei Funktionen der Presse geprägt, von der Informations- und Appellfunktion. Sie unterscheiden sich demgemäß hinsichtlich ihrer Funktionalität. Es ist aber nicht möglich, klare und feste Grenzen zwischen unterschiedlichen Textfunktionen zu ziehen; sie sollen miteinander verbunden und mit Einbeziehung der jeweiligen Textsorteneigenschaften untersucht werden. Die Neologismen in den Textsorten mit dominierender informativer Funktion vermitteln vorwiegend Tatsacheninformationen. Sie haben oft eine komplexe lexikalische Struktur (Komposita); sie sind lexikalische Informationsverdichtungen, geprägt von den medienspezifischen Eigenschaften der Tagespresse (auf möglichst engem Raum möglichst viele Informationen darzubieten). Die in den Textsorten mit dominierender Appellfunktion vorkommenden Neologismen haben nicht nur einen referentiellen, sondern auch einen stark ausgeprägten konnotativen Inhalt. Sie vermitteln Autors persönliche Einstellungen, Meinungen, positive oder negative Bewertungen; sie verstärken die Bedeutung des Bezeichneten und betonen seine Exklusivität; sie sind Euphemismen; sie sollen beim Leser Interesse erzeugen und seine Aufmerksamkeit lenken, womit sie zur Expressivität des Textes wesentlich beitragen.

Die Neologismen erweitern und bereichern den Wortschatz einer Sprache und beweisen dadurch die stark ausgeprägte sprachliche Dynamik. Trotz mancher sprachpflegerischen Kritiken dürften wir sie nicht unterschätzen, denn sie sind notwendig, sie lösen Widersprüche auf, die zwischen vorhandenem Wortschatz und neuen kommunikativen bzw. kognitiven Bedürfnissen einer Sprachgemeinschaft entstehen. Sie dürften größere Aufmerksamkeit der Sprachwissenschaftler verdienen; möglicherweise auch in der Form von periodisch erscheinenden Neologismensammlungen, denn 
die gängigen Wörterbücher können dem ständigen Zuwachs an lexikalischen Innovationen nicht gerecht werden.

\section{QUELLEN- UND LITERATURVERZEICHNIS}

\section{Quellenverzeichnis}

FRANKFURTER ALLGEMEINE ZEITUNG. Zeitung für Deutschland. (25. Sept. 1993) 223. Frankfurt am Main.

SÜDDEUTSCHE ZEITUNG. Münchner neueste Nachrichten aus Politik, Kultur, Wirtschaft und Sport. 49 (25./26. Sept. 1993) 222. München.

DIE ZEIT. Wochenzeitung für Politik, Wirtschaft, Handel und Kultur. 48 (24. Sept. 1993) 39. Hamburg.

\section{Literaturverzeichnis}

BRAUN, P. 1993. Tendenzen in der deutschen Gegenwartssprache. Sprachvarietäten. 3. Aufl. Stuttgart, Berlin, Köln.

BRINKER, K. 1988. Linguistische Textanalyse. Eine Einführung in Grundbegriffe und Methoden. 2. Aufl. Berlin.

BURGER, H. 1990. Sprache der Massenmedien. 2. Aufl. Berlin, New York.

BUBMANN, H. 1990. Lexikon der Sprachwissenschaft. 2. Aufl. Stuttgart.

CARSTENSEN, B. 1986. Beim Wort genommen. Bemerkenswertes in der deutschen Gegenwartssprache. Tübingen.

DEUTSCHE Gegenwartssprache. 1990. Tendenzen und Perspektiven. Hrsg. von G. Stickel. Berlin, New York.

DEUTSCHE Sprache. 1983. Kleine Enzyklopädie. Leipzig.

DROSDOWSKI, G. 1980. Tendenzen der deutschen Gegenwartssprache. In: Lexikon der germanistischen Linguistik. Bd. 3. Tübingen.

DUDEN Deutsches Universalwörterbuch. 1989. 2. Aufl. Mannheim, Leipzig, Wien, Zürich.

DUDEN Fremdwörterbuch. 1974. Bearbeitet von W. Müller. 3. Aufl. Mannheim, Wien, Zürich.

DUDEN Grammatik der deutschen Gegenwartssprache. 1984. Hrsg. von G. Drosdowski. 4. Aufl. Mannheim, Wien, Zürich.

EGGERS, H. 1989. Moderner Nominalstil. In: Deutsche Sprache der Gegenwart. Entwicklungen und Tendenzen. Stuttgart.

ENTWICKLUNGSTENDENZEN in der deutschen Gegenwartssprache. 1988. Hrsg. von K.-E. Sommerfeldt. Leipzig.

ERBEN, J. 1981. Neologismen im Spannungsfeld von System und Norm. In: Logos Semantikos. Festschrift für E. Coseriu, 35-43. 
FLEISCHER, W./BARZ, I. 1992. Wortbildung der deutschen Gegenwartssprache. Tübingen.

GOOD, C. 1989. Zeitungssprache im geteilten Deutschland. Exemplarische Textanalysen. München.

GREULE, A. 1980. Erbwort - Lehnwort - Neuwort. Grundzüge einer genetischen Lexikologie des Deutschen. In: Muttersprache, 263-275.

GROßE, R. 1977. Sprachliche Normen als soziale Normen und ihre Bedeutung für die Verbreitung von Neologismen. In: Linguistische Arbeitsberichte 18, 1-7.

HARTMANN, R.R.K.; STARK, F.C. 1979. Dictionary of Language and Linguistics. London.

HELLER, K. 1988. Theoretische und praktische Probleme der Neologismuslexikographie. Überlegungen und Materialien der in der Allgemeinsprache der DDR gebräuchlichen Neologismen. In: Linguistische Studien. Reihe A. Arbeitsberichte, 184. Berlin.

HERBERG, D. 1987. Stand und Aufgaben der Neologismenlexikographie des Deutschen. Vortrag, IDS-Tagung März 1987.

HERBERG, D. 1988. Neologismen - lexikologisch und lexikographisch betrachtet. In: Sprachpflege 8, 109-124.

KALZ, B. 1988. EDV-Sprache. Zu einigen Wortbildungstendenzen im Deutschen und Französischen. In: Muttersprache, 38-49.

LEXIKON sprachwissenschaftlicher Termini. 1988. Hrsg. von R. Conrad. 2. Aufl. Leipzig.

LÜGER, H.-H. 1983. Pressesprache. Tübingen.

MALETZKE, G. 1976. Ziele und Wirkungen der Massenkommunikation. Grundlagen und Probleme einer zielorientierten Mediennutzung. Hamburg.

MEYER, H.-G. 1974. Untersuchungen zum Einfluß des Englischen auf die deutsche

Pressesprache, dargestellt an zwei deutschen Tageszeitungen. In: Muttersprache, 97-134.

MOSER, H. 1959. Neuere und neueste Zeit. Von den 80er Jahren des 19. Jahrhunderts zur Gegenwart. In: Deutsche Wortgeschichte. Hrsg. von F. Maurer und F. Stroh. Berlin.

MÜLLER, W. 1976. Neue Wörter und neue Wortbedeutungen in der deutschen Gegenwartssprache. In: Universitas 8, 867-873.

MÜLLER, W. 1987. "Schlammschlacht". Schon gehört? Ein Desiderat: Das deutsche Neologismenwörterbuch. In: Sprache und Literatur in Wissenschaft und Unterricht $60,82-90$.

ORTMANN, W.D. 1979. Hochfrequente deutsche Wortformen. Bd. III. München. PRESSE und Pressewesen. 1993. Hrsg. von O. Hestermann. Stuttgart. SANDIG, B. 1986. Stilistik der deutschen Sprache. Berlin, New York. SCHIPPAN, Th. 1992. Lexikologie der deutschen Gegenwartssprache. Tübingen. SCHMITZ, U. 1987. Sprache und Massenmedien. In: Ammon, U. (Hrsg.): Sociolinguistics/Soziolinguistik. Bd.1. 821-833. 
SCHNEIDER, I. 1993. Poleznyj dialog. Journalistische Textsorten im Spiegel ihrer Schlagzeilen. München.

SEIFERT, L. 1990. Von Fremd- und Lehnwörtern und von Kunst- und anderen auserlesenen Deutungs-reichen Wörtern, oder: Darf man doch noch zu einer Elften Lobrede ansetzen? In: Deutsche Gegenwartssprache. Tendenzen und Perspektiven, 302-336.

SOWINSKI, B. 1991. Stilistik. Stiltheorien und Stilanalysen. Stuttgart.

WIRKUNGEN der Massenkommunikation. 1989. Theoretische Ansätze und empirische Ergebnisse. Hrsg. von R. Burkart. 2. Aufl. Wien.

XUEFU, D. 1987. Neologismen im heutigen Deutsch. In: Sprache und Literatur in Wissenschaft und Unterricht 59, 98-106.

Povzetek

MEDIJSKO UČINKOVITI NEOLOGIZMI V SODOBNEM NEMŠKEM JEZIKU

Magistrsko delo raziskuje neologizme, ki se pojavljajo v sodobnem nemškem publicističnem jeziku. Na osnovi važnejše literature o neologizmih je izdelana delovna definicija, ki sprejema za neologizme vse tiste besede, ki so v določenem času občutene kot nove. Gre za nove tvorjenke, ali za že znane besede, ki dobijo nov pomen, ali za besede, ki so na novo prevzete iz drugih jezikov. Raziskava upošteva 456 tako definiranih neologizmov, izpisanih iz dnevnega oz. tedenskega časopisja (Frankfurter Allgemeine Zeitung, Süddeutsche Zeitung, Die Zeit). Analizirana je njihova besednovrstna pripadnost, besedotvorna zgradba, njihov izvor in funkcije, ki jih neologizmi opravljajo v posameznih vrstah publicističnih besedil. Opravljena analiza omogoča naslednje ugotovitve: 1 . Med neologizmi prevladujejo samostalniki $(93,6 \%)$; pridevnikov $(4,6 \%)$ in glagolov $(1,8 \%)$ ni veliko. Rezultati ne presenečajo, saj publicistični jezik zahteva besedno strnjanje informacij in večjo obvestilnost besedil, to pa je mogoče doseči tudi s pogosto rabo zloženk. Novi samostalniki so pogosto rabljeni kot strokovni izrazi (v strokovnem jeziku je že zaradi značilne skladnje veliko samostalnikov), ali pa so v publicističnih besedilih zapisani namenoma, in sicer tudi v primerih, ko bi bilo možno (in je tudi ustaljeno) glagolsko izražanje. Pisci jih tvorijo in rabijo zato, ker želijo izboljšati okoliščine sporočanja in si pridobiti naslovnikovo zanimanje. 2. Približno polovica opazovanih neologizmov (43\%) so prevzete besede, ki so večinoma rabljene zato, ker so v primerjavi z obstoječimi ustreznicami pomensko močnejše ali natančnejše; izkazujejo lahko prestižnost, ali pa so jezikovno gospodarnejše. Prevladuje prevzemanje iz angleščine oz. ameriške angleščine. Prevzeti neologizmi so različno prilagojeni nemškemu jeziku, in sicer še najbolj oblikoslovno, saj večinoma pridobijo slovnične kategorije "novega" jezika, kot besedotvorne prvine pa so udeležene $\mathrm{v}$ besedotvornih postopkih. 3 . V publicističnem jeziku neologizmi podpirajo predvsem dve osnovni nalogi publicističnih besedil: informativno in apelativno, ki pa ju ni mogoče popolnoma ločevati, saj se prepletata. Enako velja za neologizme - njihove funkcije $v$ besedilu je potrebno ugotavljati celostno, in sicer tako, da upoštevamo tudi značilnosti vsakokratne besedilne vrste. $\mathrm{V}$ besedilih, ki predvsem obveščajo, neologizmi sporočajo dejstva in dogodke; v besedilih s prevladujočo apelativno funkcijo pa poleg svojega referenčnega pomena izražajo se piščeva osebna mnenja in stališča, njegove pozitivne in negativne ocene, krepijo pomen in poudarjajo enkratnost označenega. Pogosto so rabljeni tudi zato, da pri naslovniku vzbudijo zanimanje in tako povečujejo ekspresivno zaznamovanost besedila. 